\title{
Evaluation of Deep Learning for Image-based Black Pepper Disease and Nutrient Deficiency Classification
}

\author{
Choy Yuen Khew \\ Dept of Research and Quality \\ Development \\ Malaysian Pepper Board \\ Kuching, Malaysia, \\ cykhew@mpb.gov.my
}

Siaw San Hwang

School Chemical Engineering and Science

Swinburne University of Technology

(Sarawak)

Kuching Sarawak

shwang@swinburne.edu.my

\author{
Yi Qin Teow \\ Faculty of Cognitive Sciences and Human \\ Development \\ Universiti Malaysia Sarawak \\ Kota Samarahan, Malaysia \\ tyqin4237@gmail.com \\ Chih How Bong \\ Faculty of Computer Science and \\ Information Technology \\ Universiti Malaysia Sarawak \\ Kota Samarahan, Sarawak \\ chbong@unimas.my
}

\author{
Ee Tiing Lau \\ Dept of Research and Quality \\ Development \\ Malaysian Pepper Board \\ Kuching, Malaysia, \\ lau@mpb.gov.my \\ Nung Kion Lee* \\ Faculty of Cognitive Sciences and Human \\ Development \\ Universiti Malaysia Sarawak \\ Kota Samarahan, Malaysia \\ nklee@unimas.my
}

\begin{abstract}
Black pepper (Piper nigrum) diseases and nutrient deficiency can often be observed based on the symptoms exerted on its leaves. This paper aimed to investigate the effectiveness of employing a deep learning approach to classify black pepper disease and nutrient deficiency based on leaf images. We constructed a customized convolutionary neural network to determine how its training parameters would affect the prediction performances. Another two deep learning neural networks VGG16 and Inception V3, are also employed for comparisons. We have sampled 947 images from farms in Sarawak consisted of 8 classes in total. Image augmentation is performed on the images to produce a total of 9532 images. The result shows that the customized CNN performed slightly better than the other two deep learning approaches at a 0.98 sensitivity rate. Furthermore, image augmentation contributed to improving prediction performance for all the deep learning models. This study has demonstrated that deep learning is a feasible approach for classifying black pepper diseases and nutrient deficiency based on leaf images.
\end{abstract}

Keywords-deep learning, black pepper, plant disease, convolutionary neural networks

\section{INTRODUCTION}

Black pepper is known as the King of Spices and is widely used for culinary and food seasoning purposes. Global demand for black pepper has steadily increased over the last decade, which amounted to a value of $4.1 \mathrm{~b}$ US dollars in 2018 [1]. Growing black pepper is challenging because it is a high nutrient-demanding crop and easily affected by diseases. In Malaysia, the yield losses due to the conditions have accounted for about 25 per cent of the country's pepper production [2]. The pepper farmers face significant constraints in managing both diseases and nutrient deficiency in black pepper cultivation. The delay in recognizing pepper plants' health problems and late intervention to control the disorder further exacerbated the plight of pepper farmers.

Most of Malaysia's pepper farms are grown by smallholder farmers and are located in hilly rural areas. Any plant problems that require advisory assistance are still applied through conventional means that need farmers to notify local pepper government agency (Malaysian Pepper Board, MPB) to receive advisory services from extension officers.
However, the limited number of extension staff and scattered distribution of pepper farms led to the extension services' efficacy become another question. A recent outbreak of foot rot diseases in Sarawak in 2018 has caused severe damage to pepper farms in a short period (as shown in Fig 1). That was mainly due to the delay in detection and late intervention of the infected pepper farms. The incidence indicated the critical need for extension services in the early intervention of the plant problem. Besides, efficient extension services also ensure the transfer of knowledge and technology to farmers to help them make the right decisions to mitigate crop problems.

We can visually observe some diseases and nutrient deficiency through different plant parts, such as leaves, branches, or berries. Trained farmers or plant experts can identify the plant health issues based on the visual inspection because the infection patterns are consistent with texture colour and infected areas on the plant parts. Hence, computer vision techniques based on digital device acquired images can recognize black pepper diseases.

Previous studies on black pepper disease classification have focused on feature engineering, image processing, and algorithm design for black pepper classification [3]. Nevertheless, there has not been any study using a deep learning approach for black pepper health issues classification.

This paper evaluates the effectiveness of using the deep learning approach, namely convolutionary neural networks, for automated black pepper health issues classification based on leaf images. Previous studies have shown accuracy rates of $80-90 \%$ in black pepper disease recognition. Therefore, it is interesting to investigate the effectiveness of the deep learning approach image-based classification of black pepper plant issues. This study is an initial step towards determining the efficacy of a deep learning model deployed in mobile apps to diagnose black pepper disease and nutritional disorders rapidly.

The following is an overview of the structure of this publication. The work-related to using deep learning for plant issue diagnostics are presented in the next section. The approach, which involves data collection, data pre-processing

This work was supported by the Kementerian Sains, Teknologi dan Inovasi, under the SMARTFUND grant, project PR1118A1278 and by a grant from the Malaysian Pepper Board (P20113000010025). *Corresponding author 